\title{
Main Differentiating Issues in Aligning Information Systems with Business Performance
}

Silvia C. Fernandes, Research Centre for Spatial and Organizational Dynamics (CIEO), Faculty of Economics, University of Algarve, Campus de Gambelas, Faro, Portugal

\begin{abstract}
The information systems and technologies (IS/IT) are a decisive platform in the responsecapacity of organizations to the new management imperatives through new solutions that permit to improve their competitive position. As information management is a highly differentiating area, the present study compares several enterprises of different sectors, located (headoffice or branch) in the Algarve region, with the aim of systematising the main differentiating issues related with adoption, restructuring and implementation of information systems and supportive technologies. Every organization should define an accurate and in time plan which, in case of adopting and implementing information systems, has to analyse results within the same plan and on a yearly basis of the system 's activity always aligned with the business strategy. Given the potential of ERP and CRM as powerful tools to guide customer-oriented understanding, greater attention is required on their economic viability for sustainable business dynamics.
\end{abstract}

Keywords: $\quad$ Business Performance, Customer Relationship Management, Enterprise Resource Planning, Information Systems, Process Integration, Strategic Alignment

\section{INTRODUCTION}

Modern drivers of change have led to new features for sustaining business competitiveness: virtual enterprises, creative design, interactivity, mobile platforms, etc. These responses require IT-based tools, the increase of information content and creative teams. Any enterprise that wants to optimise its success in the information society must have a basic awareness and a strategy for dealing with this new environment. With the shortening of life cycle and time to market this has to evolve with a pace that approaches a need for its real time creation (Philipson, 2008). For example, the internet brings critical new functionalities to enterprises by real time business dynamics and relations. If they don't constantly improve the process of managing this, they would quickly become paralysed. Building custom software systems to accomplish it can become so complex that the software requires an 'army' of engineers and consultants to manage it. Small to medium businesses need a way to organise their data 
on a smaller scale and without the expense of hiring costly consultants or paying for maintenance contracts. The approach of building and managing a custom software solution is financially out of reach for small to medium sized companies. Therefore companies are moving to web-based software as it can eliminate the need to purchase and manage computer servers, firewalls and software servers just to run the application. A broadband connection to the internet is recommended for sustaining a design in a very modular way, which allows for tremendous flexibility in building intranets that meet the needs of a particular activity. The applications built in a modular way can be combined to form an application suite which is best used as a company intranet. Modules can be mixed and matched to meet a particular need as they keep the intranet free from clutter because firms configure just the features they wish in their intranets.

Many firms are investing in the implementation of IT-based tools to enhance their service, interactivity and tracking. The present paper emphasises the business potential of ERP (Enterprise Resource Planning) systems together with new functionalities more oriented to link front-end management with the ERP system (back-end). These can include CRM (Customer Relationship Management) and CMS (Content Management) functions as an integrated approach to identifying, acquiring and retaining customers. Among several tools that firms apply on their websites and intranets are CRM and CMS. The first enables organizations to manage customer interactivity across multiple channels or business lines and maximize the value of every customer interaction. CRM is a strategy used to learn more about customers' needs and behaviours in order to develop stronger relationships with them. CRM features range from advanced applications, such as database-driven product customization tools, to simple ones as a line of contact information on a web page.

Combined with CRM, the CMS features enable building powerful applications with a very high level of content organization, so that many competitive aspects including efficiency and flexibility are achieved. CMS contributes to enhance the potential of personalization that these features can entail as their focus is on content management, which combines technology and business processes to effectively deliver large amounts of diverse information to different media (Forsyth, 2004). As an increasing number of new businesses specially in service sectors are online, MC (Mass Customisation) features are joining these tools. The study of Sigala (2010) underlines the importance of IS design and customer-focused approaches for supporting MC services. This kind of functionalities has revolutionized front-end effective management and its integrative link with backend business processes. This involves a holistic approach in order to match businesses to what partners and customers want by providing the dynamic creation, distribution and publishing of information.

\section{ACTING CREATIVELY AND DECISIVELY}

For many entrepreneurs and managers, seeing an opportunity is the easy part. The real challenge lies in seizing it (Basadur, 2009). Those who have identified a gap in the market often stumble because they can't scale their organization to meet booming customer demand before competitors encroach. The rapid growth required to seize an opportunity places enormous strains on a company's resources, organization and management. Basadur (2009), founder of the enterprise Basadur Applied Creativity, defends that a common mistake among both start-ups and established companies is to overcommit resources before a business plan has been nailed down.

This entrepreneur drives attention to the strengths of the individual's thinking style and to a problem solving system to create measurable and successful solutions. He believes that key ingredients of quality results must include the right people with the right knowledge, in a process using knowledgeable skills and tools. The keys are adaptability, constant re-invention 
11 more pages are available in the full version of this document, which may be purchased using the "Add to Cart" button on the product's webpage:

www.igi-global.com/article/main-differentiating-issuesaligning-information/78934

\section{Related Content}

An Approach to Deploying SOA in Technological Information Integration: A Case Study

Zhuofeng Zhao, Haifang Fu and Jun Fang (2010). International Journal of Service

Science, Management, Engineering, and Technology (pp. 1-18).

www.igi-global.com/article/approach-deploying-soa-technological-

information/43615

Location-Based Services (LBS) in Micro-Scale Navigation: Shortcomings and Recommendations

Suleiman Almasri, Muhammad Alnabhan, Ziad Hunaiti and Eliamani Sedoyeka

(2011). Developing Technologies in E-Services, Self-Services, and Mobile

Communication: New Concepts (pp. 234-252).

www.igi-global.com/chapter/location-based-services-lbs-micro/54966

A Study on Relationships Among Software Engineering Capabilities in Japan Using Panel Analysis

Yasuo Kadono (2011). International Journal of Service Science, Management,

Engineering, and Technology (pp. 20-32).

www.igi-global.com/article/study-relationships-among-software-

engineering $/ 59880$

Performance Analysis of Cloud Computing Centers for Bulk Services

Veena Goswami, Sudhansu Shekhar Patra and G. B. Mund (2012). International

Journal of Cloud Applications and Computing (pp. 53-65).

www.igi-global.com/article/performance-analysis-cloud-computing-

centers/75116 